\title{
Correction: Dapagliflozin add-on to metformin in type 2 diabetes inadequately controlled with metformin: a randomized, double-blind, placebo-controlled 102-week trial
}

Clifford J Bailey ${ }^{1 *}$, Jorge L Gross ${ }^{2}$, Delphine Hennicken ${ }^{3}$, Nayyar lqbal ${ }^{4}$, Traci A Mansfield ${ }^{4}$ and James F List ${ }^{4}$

\section{Correction}

The authors [1] note that the standard errors in Table 3 (Table 1 here) are actually standard deviations. The reference to standard errors in the footnote has now been edited accordingly.

This is a Correction article on http://www.biomedcentral. com/1741-7015/11/43.

\footnotetext{
* Correspondence: c.j.bailey@aston.ac.uk

1 Life and Health Sciences, Aston University, Birmingham B4 7ET, UK

Full list of author information is available at the end of the article
} 
Table 1 Summary of laboratory parameters

\section{Sodium (mmol/L)}

PBO + MET

$(n=137)$

$\mathrm{n}$

Baseline

Change at week 102

$P$ vs PBO + MET

Potassium (mmol/L)

n

Baseline

Change at week 102

$P$ Vs PBO + MET

Serum creatinine ( $\mu \mathrm{mol} / \mathrm{L}$ )

$\mathrm{n}$

Baseline

Change at week 102

$P$ VS PBO + MET

Serum uric acid $(\mu \mathrm{mol} / \mathrm{L})^{a}$

$\mathrm{n}$

Baseline

Change at week 102

$P$ vs PBO + MET

Blood urea nitrogen ( $\mathrm{mmol} / \mathrm{L})$

n

Baseline

Change at week 102

$P$ Vs $\mathrm{PBO}+\mathrm{MET}$

Haematocrit (\%)

n

Baseline

Change at week 102

$P$ vs $\mathrm{PBO}+\mathrm{MET}$

\section{Haemoglobin (g/L)}

$n$

Baseline

Change at week 102

$P$ vs PBO + MET

Systolic blood pressure $(\mathrm{mm} \mathrm{Hg})$

n

Baseline

Change at week 102

$P$ vs PBO + MET
72

$138.9(2.3)$

$-0.04(2.7)$

72

4.35 (0.47)

$-0.08(0.55)$

72

76.9 (17.9)

$-0.9(10.3)$

28

$314(79)$

-1.78 (54.13)

72

$5.33(1.55)$

0.56 (1.24)

71

42.61 (3.90)

$-1.43(3.29)$

71

$142.8(13.45)$

$-4.9(10.14)$

72

128 (15)

1.5 (13.7)
DAPA $2.5 \mathrm{mg}+$ MET

( $n=137)$

77

138.5 (2.9)

$0.2(2.8)$

0.1434

77

$4.34(0.46)$

$-0.01(0.45)$

0.3366

77

78.7 (18.1)

$-1.8(9.7)$

0.9954

35

322 (80)

$-55.9(50.32)$

0.0054

77

$5.51(1.41)$

0.70 (1.54)

0.2299

78

42.38 (3.99)

0.84 (2.53)

$<0.0001$

78

142.5 (14.25)

1.5 (7.73)

$<0.0001$

78

127 (14)

0.7 (16.1)

0.1111
DAPA $5 \mathrm{mg}+$ MET

$(n=137)$

87

$138.6(2.3)$

0.1 (2.6)

0.1905

87

4.37 (0.39)

$-0.05(0.35)$

0.4563

88

79.6 (19.3)

-3.5 (12.7)

0.4720

47

323 (88)

-46.4 (64.36)

0.0013

88

5.65 (1.81)

$0.42(1.87)$

0.4169

87

42.15 (3.59)

1.35 (2.48)

$<0.0001$

87

141.6 (12.19)

$3.1(8.40)$

$<0.0001$

88

127 (14)

$-1.1(13.2)$

0.0136
DAPA $10 \mathrm{mg}+$ MET $(n=135)$

93

$139.1(2.2)$

0.1 (2.6)

0.3978

93

$4.37(0.43)$

$-0.07(0.47)$

0.9918

93

77.8 (17.8)

$-2.7(10.6)$

0.9032

57

323 (80)

-52.9 (64.36)

0.0002

93

$5.34(1.41)$

0.73 (1.44)

0.0845

93

42.88 (3.95)

1.54 (2.78)

$<0.0001$

94

143.6 (13.10)

4.1 (8.84)

$<0.0001$

94

126 (16)

$-0.3(15.0)$

0.0067 
Table 1 Summary of laboratory parameters (Continued)

\section{Diastolic blood pressure $(\mathrm{mm} \mathrm{Hg})$}

n

Baseline

7278

88

94

Change at Week 102

$81(9) \quad 80(9)$

$81(9)$

Change at Week 10

$-1.0(7.9) \quad-0.1(8.1)$

$-1.5(8.1)$

Data are means (SD) at baseline or mean changes (SD) from baseline at week 102 including data after rescue unless noted. $\mathrm{n}$ is the number of treated patients with non-missing baseline and Week 102 values for the specific test. ${ }^{a}$ Excluding data after rescue. Abbreviations: $S D$ standard deviation, $S E$ standard error, $P B O$ placebo, MET metformin, DAPA dapagliflozin.

\section{Author details}

${ }^{1}$ Life and Health Sciences, Aston University, Birmingham B4 7ET, UK. ${ }^{2}$ Endocrine Division, Universidade Federal do Rio Grande do Sul, Avenida Paulo Gama 110-Reitoria, Porto Alegre 90040-060, Brazil. ${ }^{3}$ Global Biometric Services, Bristol-Myers Squibb, Parc de l'Alliance, Avenue de Finlande 4, Braine I'Alleud B-1420, Belgium. ${ }^{4}$ Global Clinical Research, Bristol-Myers Squibb, Route 206 \& Province Line Road, Princeton, NJ 08543, USA.

Received: 20 August 2013 Accepted: 20 August 2013

Published: 2 September 2013

\section{Reference}

1. Bailey CJ, Gross JL, Hennicken D, lqbal N, Mansfield TA, List JF: Dapagliflozin add-on to metformin in type 2 diabetes inadequately controlled with metformin: a randomized, double-blind, placebo-controlled 102-week trial. BMC Medicine 2013, 11:43.

doi:10.1186/1741-7015-11-193

Cite this article as: Bailey et al:: Correction: Dapagliflozin add-on to metformin in type 2 diabetes inadequately controlled with metformin: a randomized, double-blind, placebo-controlled 102-week trial. BMC Medicine 2013 11:193.

\section{Submit your next manuscript to BioMed Central and take full advantage of:}

- Convenient online submission

- Thorough peer review

- No space constraints or color figure charges

- Immediate publication on acceptance

- Inclusion in PubMed, CAS, Scopus and Google Scholar

- Research which is freely available for redistribution 